\title{
TRANSPLANTE CARDÍACO: COMPLICAÇÕES APRESENTADAS POR PACIENTES DURANTE A INTERNAÇÃO
}

\author{
Heart Transplantation: complications presented by patients during the hospitalization \\ Trasplante de corazón: complicaciones que presentan los pacientes durante la \\ hospitalización
}

\author{
Elaine Araújo da Silva ${ }^{1}$ \\ Daclé Vilma Carvalho
}

\section{RESUMO}

Com o avanço da ciência, a insuficiência cardíaca tem como possibilidade de intervenção o transplante cardíaco. Este trabalho teve como objetivos traçar o perfil demográfico dos pacientes submetidos ao transplante cardíaco e identificar as complicações apresentadas durante a internação. Trata-se de um estudo descritivo, quantitativo, realizado com 44 prontuários de pacientes submetidos a transplante de junho/2006 a julho/2009 em um Hospital Universitário de Belo Horizonte, Minas Gerais, Brasil. 0 projeto atendeu às recomendações da Resolução 196/96. Pelos resultados, a maioria dos pacientes era do sexo masculino, casada, escolaridade de nível fundamental e idade média de 45,2 anos. A miocardiopatia chagásica foi a principal causa de insuficiência cardíaca. As complicações predominantes foram alteração da glicemia, insuficiência renal e infecções. A taxa de mortalidade na internação foi de $20,4 \%$. Além das complicações biológicas só foram registradas ansiedade e depressão. São necessárias pesquisas sobre o tema nos âmbitos psicossociais e espirituais, contribuindo para o desenvolvimento científico.

Palavras-chave: Enfermagem. Transplante de coração. Transplante de órgãos. Complicações pós-operatórias.

\begin{abstract}
Nowadays, with advance in technology and medicine the last alternative of treatment to heart failure is the cardiac transplantation. This work was developed with the following objectives to delineate the demographic profile of patients undergoing heart transplantation and complications presented by them after this procedure in the hospitalization. This is a descriptive and quantitative study conducted with the 44 records of patients undergoing heart transplantation from June/2006 to July/2009, in a University Hospital in Belo Horizonte, Minas Gerais, Brazil. The project attended to Resolution 196/96 recommendations. According to the results the majority of patients were male, married, with elementary education and the mid age of 45.2 years. The Chagasic cardiomyopathy was the main cause of heart failure. Complications that prevailed during the admission were alteration in blood glucose, kidney failure and infections. The mortality rate was $20.4 \%$ during hospitalization. In addition to biological complications were recorded only anxiety and depression. It is necessary to research on this subject in the psychosocial and spiritual spheres, contributing to the scientific development.
\end{abstract}

Keywords: nursing. heart transplantation. organ transplantation. postoperative complications.

\section{Resumen}

Hoy día, con los avances de la medicina y de la tecnología para el tratamiento de la insuficiencia cardiaca irreversible, se ha utilizado como última intervención el trasplante cardíaco. Este trabajo se desarrolló con los objetivos de delinear el perfil demográfico de los pacientes sometidos a trasplante cardíaco e identificar las complicaciones presentadas por ellos después del trasplante de corazón. Estudio descriptivo y cuantitativo, realizado en conjunto con los registros de 44 pacientes sometidos a trasplante de corazón desde Jun/2006 hasta Jul/2009, en un Hospital Universitario de Belo Horizonte, Minas Gerais, Brasil. El proyecto cumplió con las recomendaciones de la Resolución 196/96. Los resultados mostraron que la mayoría de los pacientes eran del sexo masculino, casados, con educación primaria y edad media de 45,2 años. Miocardiopatía chagásica fue la principal causa de insuficiencia cardíaca. Complicaciones que prevalecieron durante el ingreso fueron la alteración de la glucosa en la sangre, insuficiencia renal y las infecciones. La tasa de mortalidad fue del $20,4 \%$ durante la hospitalización. Además de las complicaciones biológicas, se registraron también ansiedad y depresión. Se hace necesario investigaciones sobre el tema en los ámbitos psicosociales y espirituales, contribuyendo al desarrollo científico.

Palabras clave: enfermería. trasplante de corazón. trasplante de órganos. complicaciones postoperatorias.

\footnotetext{
'Mestre em enfermagem pela Escola de Enfermagem - Universidade Federal de Minas Gerais (UFMG). Enfermeira coordenadora técnica assistencial Hospital das Clínicas (UFMG). Coordenadora suplente da residência multiprofissional do Hospital das Clínicas (UFMG) - área cardiovascular. Belo Horizonte - MG. Brasil. E-mail: elainearaujos@gmail.com; ${ }^{2}$ Doutora em Enfermagem pela Universidade de São Paulo (USP). Professora Associada da Escola de Enfermagem da Universidade Federal de Minas Gerais - Departamento de Enfermagem Básica. Belo Horizonte - MG. Brasil. E-mail: dacle@enf.ufmg.br
} 


\section{INTRODUÇÃO}

As doenças do aparelho circulatório constituem um grupo de doenças crônicas que é considerado um grande problema atual de saúde pública. Dentre essas, as doenças cardiovasculares aparecem em primeiro lugar como responsáveis pela mortalidade de 17.528 pessoas em um total global de 35.576 mortes no mundo ${ }^{1}$.

Estudos mostram que, nos últimos anos, o número de acometimento por essas doenças vem crescendo em ritmo acelerado não somente em países desenvolvidos, mas também nos países em desenvolvimento. Essas projeções indicam 0 aumento de sua importância relativa em países de baixa e média renda².

O Brasil, além do aumento da mortalidade por doenças cardiovasculares, as internaç̃es pelo Sistema Único de Saúde (SUS) vêm crescendo devido à ascensão progressiva desse grupo de doenças, que, a partir de 2003, foi responsável pelo maior custo com as internações com destaque para insuficiência cardíaca ${ }^{3}$.

As instituições de saúde têm procurado se reestruturar para atender a demanda crescente das doenças cardiovasculares e investem em unidades cardiológicas, procurando aprimorar tecnologias para intervirem de maneira eficaz.

Com os avanços da medicina e da tecnologia, os casos graves, antes considerados irreversíveis, têm o recurso de cirurgias para substituição de estruturas cardíacas como válvulas e até do próprio órgão, como nos transplantes cardíacos.

0 primeiro transplante cardíaco ocorreu na África, em 1967. No Brasil, sua primeira ocorrência foi em $1968^{4}$. Em Minas Gerais, esse procedimento ocorreu, pela primeira vez, em 1986, na cidade de Belo Horizonte ${ }^{5}$ e vem se desenvolvendo de forma crescente ao longo das últimas décadas. Em 2010, Minas Gerais tornou-se o segundo maior centro transplantador de coração do país. Destaca-se que, atualmente, em Minas Gerais, duas instituições têm realizado transplantes cardíacos.

0 transplante cardíaco é considerado uma cirurgia de grande porte e de alta complexidade e pode apresentar, além de complicações inerentes a qualquer tipo de cirurgia, outras de naturezas biológica, psicológica, social, espiritual. Portanto, exige assistência de equipe multiprofissional com capacitação específica ${ }^{6}$.

Estudos apontam algumas complicações decorrentes do transplante cardíaco, tais como: insuficiência renal, sangramento, rejeição, bloqueio átrio ventricular total (BAVT) permanente, convulsão, débito cardíaco baixo, lesão neurológica e até mesmo reativação do protozoário Trypanossoma cruziem pacientes chagásicos ${ }^{7}$.

Em estudo realizado com 49 pacientes internados em CTI, em pós-operatório de transplante cardíaco, foram identificados problemas como sentimento de culpa, ansiedade, manifestações de baixa autoestima, entre outros ${ }^{5}$.

A equipe de enfermagem, pela assistência integral e ininterrupta a estes pacientes, desempenha papel fundamental ${ }^{6}$, principalmente no pós-operatório imediato e mediato. Por meio da observação direta e contínua, tem oportunidade de detectar precocemente sinais de possíveis complicações, o que possibilita a rápida ação dos profissionais de saúde no sentido de preveni-las ou pelo menos minimizá-las.

Por se tratar de um procedimento recente no hospital universitário, ainda não há conhecimento sistematizado das características dos pacientes que são submetidos às cirurgias de transplante cardíaco, bem como das complicações apresentadas por eles.

O conhecimento de complicações que comumente ocorrem em pós-operatório de transplante cardíaco subsidiará o planejamento da assistência, principalmente em relação a medidas de prevenção ou detecção precoce de alterações que sinalizem o seu início, evitando o seu surgimento ou agravamento.

Essa pesquisa teve como objetivos: traçar o perfil demográfico dos pacientes submetidos a transplante cardíaco e identificar complicações apresentadas pelos pacientes após o transplante cardíaco durante internação.

\section{MÉTODO}

Trata-se de um estudo descritivo e exploratório, escolhido pela possibilidade de se descrever os fenômenos, e não de se estabelecer correlações ou verificar a frequência probabilística da ocorrência deles.

0 estudo foi realizado em um hospital universitário em Belo Horizonte que é referência nos sistemas estadual e municipal no atendimento de média a alta complexidade. Entre os serviços de alta complexidade, estão os transplantes. Dos já realizados (medula óssea, rim, fígado, pulmão, duplo transplante de pâncreas-rim), iniciou-se em junho de 2006 o transplante cardíaco.

A população foi constituída por todos os 53 pacientes submetidos a transplante cardíaco, no período de 29 de junho de 2006 a 31 de julho de 2009. De acordo com os critérios, foram excluídos prontuários de três pacientes que faleceram no transoperatório, de dois que não autorizaram o uso de seus dados e dos quatro que não devolveram o Termo de Consentimento Livre Esclarecido (TCLE) assinado embora tenham concordado verbalmente com a participação no estudo. Assim, a amostra estudada foi constituída por 44 prontuários de pacientes, correspondendo a 83,0\% da população.

A coleta de dados foi realizada pelas próprias autoras, nos prontuários dos pacientes após assinatura do TCLE, utilizando um formulário com itens para identificar: Sexo, Estado civil, Faixa etária, Profissão, Religião, Tempo de pós-operatório, Hábitos (tabagismo, uso de álcool e outras drogas ilícitas), Etiologia de base da IC, Comorbidades e Complicações pósoperatórias.

Os dados foram digitados em uma planilha confeccionada no software Microsoft Excel 11.0 pela técnica de dupla digitação, 
e foi utilizado o autofiltro para identificar e corrigir suas discrepâncias. Foi utilizado o programa estatístico Statistics Package for Social Science (SPSS), versão 18.

0 projeto foi aprovado pelo Comitê de Ética em Pesquisa da Universidade Federal de Minas Gerais (COEP) UFMG), parecer ETIC n ${ }^{0}$ 0632-09. Todas as recomendações para pesquisas que envolvem seres humanos foram atendidas em consonância com a Resolução 196/96, do Conselho Nacional de Saúde (CNS).

\section{RESULTADOS}

A maioria dos pacientes transplantados de coração $(81,8 \%)$ era do sexo masculino. A idade variou entre 21 e 64 anos, e a maioria deles tinha mais de 40 anos $(70,5 \%)$. Chama a atenção o percentual $(29,5 \%)$ de pacientes jovens, de 21 a 40 anos. A maioria dos pacientes era casada $(77,3 \%)$.

Quanto à religião, a falta de registro foi detectada na maioria $(65 \%)$ dos prontuários. Portanto, para o cálculo, consideramos apenas os 15 prontuários que continham esta informação, identificando-se que a maioria dos pacientes era evangélica $(60,0 \%)$, seguidos por católicos $(33,3 \%)$.

Em 45,5\% prontuários, não havia registro da escolaridade do paciente. A maioria dos que tinham tal informação tinha apenas o ensino fundamental (62,5\%). A maioria $(56,8 \%)$ dos pacientes desempenhavam atividades que não exigiam grau de instrução ou ensino fundamental, tais como ajudante de carga, açougueiro, auxiliar de serviços gerais, costureiro, faxineiro, trabalhador rural, entre outras.

Com relação à moradia, a maioria $(52,3 \%)$ dos pacientes tinha registro de Belo Horizonte $(\mathrm{BH})$ ou da Região Metropolitana de Belo Horizonte e 47,7\% do interior de MG. Estes dados podem não corresponder exatamente à realidade, pois, mesmo residindo em outro município ou estado, o paciente precisava ter um endereço em BH.

A insuficiência cardíaca é a doença de base que leva ao transplante cardíaco. A sua classificação, segundo as causas, está apresentada na Tabela 1.

Tabela 1 - Classificação da insuficiência cardíaca, segundo a causa, apresentada por pacientes submetidos ao transplante cardíaco em um hospital universitário, no período de jun/2006-jul/2009. Belo Horizonte, 2011.

\begin{tabular}{lc}
\hline \multicolumn{1}{c}{ Causa da Insuficiência Cardíaca } & \% \\
\hline Miocardiopatia chagásica & 50 \\
Miocardiopatia dilatada idiopática & 20,5 \\
Miocardiopatia isquêmica & 15,9 \\
Miocardiopatia de outras etiologias & 13,6 \\
\hline Total & 100 \\
\hline
\end{tabular}

A miocardiopatia chagásica foi identificada em 50,0\% dos pacientes, seguida pela miocardiopatia dilatada idiopática $(20,5 \%)$ e pela miocardiopatia isquêmica $(15,9 \%)$. Na categoria de Miocardiopatias de outras etiologias (13,6\%) foram agrupadas as miocardiopatias hipertensiva, restritiva, valvar e periparto.

Tabela 2 - Distribuição dos pacientes submetidos a transplante cardíaco, em um hospital universitário, no período de jun/2006-jul/2009, segundo o tempo de internação e desfecho. Belo Horizonte, 2011.

\begin{tabular}{lcccccc}
\hline \multicolumn{1}{c}{ DP0 } & & Total & & & Alta & \multicolumn{3}{c}{ Óbito } \\
\hline & $\mathbf{n}$ & $\mathbf{\%}$ & $\mathbf{N}$ & $\mathbf{\%}$ & $\mathbf{n}$ & $\boldsymbol{\%}$ \\
$<3$ & 1 & 2,3 & - & - & 1 & 2,3 \\
$4-6$ & 2 & 4,5 & - & - & 2 & 4,5 \\
$7-9$ & - & - & - & - & - & - \\
$10-12$ & 1 & 2,3 & 1 & 2,3 & - & - \\
$13-15$ & 4 & 9,0 & 2 & 4,5 & 2 & 4,5 \\
$16-18$ & 3 & 6,8 & 3 & 6,8 & - & - \\
$19-21$ & 5 & 11,4 & 3 & 6,8 & 2 & 4,5 \\
$22-31$ & 11 & 25,0 & 10 & 22,7 & 1 & 2,3 \\
$32-40$ & 7 & 16,0 & 7 & 16,0 & - & - \\
$43-48$ & 4 & 9,0 & 4 & 9,0 & - & - \\
$52-101$ & 6 & 13,7 & 5 & 11,4 & 1 & 2,3 \\
\hline Total & 44 & 100 & 35 & 79,5 & 9 & 20,4 \\
\hline
\end{tabular}


0 período internação variou de 1 a 101 dias após a cirurgia e foi categorizado segundo o desfecho, em intervalos de dias. Mais da metade dos pacientes $(54,5 \%)$ ficou internada de 10 a 31 dias e, desses, 20,8\% faleceram.
Dos $38,6 \%$ de pacientes que estiveram internados por um período que variou de 32 a 101 dias, apenas um morreu. Destaca-se que dos $6,8 \%$ de pacientes que ficaram internados no máximo até 6 dias, todos faleceram neste intervalo.

Tabela 3 - Distribuição dos pacientes submetidos ao transplante cardíaco em um hospital universitário, no período de jun/2006-jul/2009, segundo as complicações apresentadas por dia de pós-operatório. Belo Horizonte, 2011.

\begin{tabular}{|c|c|c|c|c|c|c|c|c|c|c|c|}
\hline \multirow{3}{*}{ Complicaçōes } & \multicolumn{11}{|c|}{ DPO } \\
\hline & \multirow[t]{2}{*}{$1-3$} & \multirow[t]{2}{*}{$4-6$} & \multirow[t]{2}{*}{$7-9$} & \multirow[t]{2}{*}{$10-12$} & \multirow[t]{2}{*}{ 13-15 } & \multirow[t]{2}{*}{$16-18$} & \multirow[t]{2}{*}{$19-21$} & \multirow[t]{2}{*}{$22-31$} & \multirow[t]{2}{*}{$32-40$} & \multicolumn{2}{|c|}{ Total } \\
\hline & & & & & & & & & & n & $\%$ \\
\hline Alteração da glicemia & 35 & - & - & - & - & 1 & - & - & - & 36 & 13,7 \\
\hline Insuficiência renal & 13 & 6 & 2 & 3 & 1 & - & - & 1 & - & 26 & 9,9 \\
\hline Infecçōes & 4 & 3 & 3 & 4 & 2 & - & 2 & 2 & 3 & 23 & 8,8 \\
\hline Rejeição & 1 & - & 3 & 8 & 5 & 1 & 1 & 1 & - & 20 & 7,6 \\
\hline Disfunção do enxerto & 7 & 4 & 1 & 4 & - & - & - & - & - & 16 & 6,1 \\
\hline $\begin{array}{l}\text { Distúrbios } \\
\text { gastrointestinais }\end{array}$ & - & - & 1 & 3 & - & 2 & - & 7 & 1 & 14 & 5,3 \\
\hline $\begin{array}{l}\text { Sangramento } \\
\text { aumentado }\end{array}$ & 13 & - & - & - & - & - & - & - & - & 13 & 5,0 \\
\hline $\begin{array}{l}\text { Hipertensão arterial } \\
\text { sistêmica }\end{array}$ & 3 & 2 & 2 & 1 & - & 1 & 1 & 3 & - & 13 & 5,0 \\
\hline Cefaleia & 1 & 2 & 3 & 1 & - & 3 & 1 & 2 & - & 13 & 5,0 \\
\hline Arritmias & 5 & 2 & - & - & 1 & 1 & - & 1 & 1 & 11 & 4,2 \\
\hline $\begin{array}{l}\text { Lesões/manchas na } \\
\text { pele }\end{array}$ & 4 & - & 2 & - & 2 & - & - & - & 3 & 11 & 4,2 \\
\hline Choque & 2 & 1 & - & 1 & 1 & 1 & 1 & 3 & - & 10 & 3,8 \\
\hline Sepse & 1 & 1 & - & 3 & 2 & - & - & 1 & 2 & 10 & 3,8 \\
\hline $\begin{array}{l}\text { Tamponamento } \\
\text { cardiaco }\end{array}$ & 2 & 1 & - & 2 & - & 1 & - & - & 1 & 7 & 2,7 \\
\hline Derrame pericárdico & - & 1 & 3 & - & 2 & - & - & 1 & - & 7 & 2,7 \\
\hline Convulsão & 3 & - & 1 & 1 & - & 2 & - & - & - & 7 & 2,7 \\
\hline $\begin{array}{l}\text { Alterações do } \\
\text { humor/personalidade }\end{array}$ & - & 1 & - & 2 & - & - & - & 3 & - & 6 & 2,3 \\
\hline $\begin{array}{l}\text { Encefalopatia } \\
\text { delirium }\end{array}$ & 1 & 1 & - & - & 1 & - & - & 3 & - & 6 & 2,3 \\
\hline Outras & 1 & 1 & 1 & 2 & 1 & 2 & - & 5 & - & 13 & 4,9 \\
\hline \multirow[t]{2}{*}{ Total } & 96 & 26 & 22 & 35 & 18 & 15 & 6 & 33 & 11 & 262 & 100,0 \\
\hline & $36,6 \%$ & $10,0 \%$ & $8,4 \%$ & $13,3 \%$ & $6,9 \%$ & $5,7 \%$ & $2,3 \%$ & $12,6 \%$ & $4,2 \%$ & & \\
\hline
\end{tabular}

Nota: DPO - dia de pós-operatório

Durante a internação, no período pós-operatório fez-se o registro de 262 complicações que foram identificadas no prontuário dos pacientes. Embora alguns pacientes tenham apresentado a mesma complicação mais de uma vez, esta foi computada apenas na sua primeira ocorrência.

As complicações identificadas foram agrupadas em 19 categorias: Alteração da glicemia, Insuficiência renal, Infecções, Rejeição do enxerto cardíaco, Disfunção do enxerto cardíaco, Distúrbios gastrointestinais, Sangramento aumentado, Hipertensão arterial sistêmica, Cefaleia, Arritmias, Choque, Sepse, Tamponamento cardíaco, Derrame pericárdico, Convulsões, Lesões/manchas na pele, Alteração do humor ou da personalidade, Encefalopatia ou delirium, e Outras. Nesta última categoria foram agrupadas as complicações apresentadas somente por até 3 pacientes.
Tendo em vista o número de complicações (262) e o número de pacientes (44), tem-se, em média, 5,9 complicações por paciente. 0 maior número de complicações apresentadas pelos pacientes na fase hospitalar predomina até $013^{\circ}$ dia. Após, inicia-se um declínio consideravelmente a partir do $19^{\circ} \mathrm{DPO}$. Após $031^{\circ}$ dia do transplante apenas três pacientes apresentaram infecção; outros três, lesão na pele; um, arritmia; e outro, tamponamento cardíaco.

As complicações que predominaram em mais de 50,0\% dos pacientes são alteração da glicemia, insuficiência renal e infecções. Infecções, choque, rejeição do enxerto, hipertensão e cefaleia ocorreram praticamente em todo o primeiro mês.

$\mathrm{Na}$ categoria Outras estão incluídas as seguintes complicações: neuropatia periférica (1), dislipidemia (1), 
diabetes mellitus (1), intolerância ao imunossupressor (2), bacteremia (2), gota agudizada (3) e acidente vascular encefálico (3).

\section{DISCUSSÃO}

Confirmando o que ocorre em outros cenários nacionais e internacionais, em nosso estudo, a maioria dos pacientes submetidos ao transplante cardíaco foi do sexo masculino $(81,8 \%)$.

No Brasil, em estudos realizados em Pernambuco ${ }^{8}$, São Paulo ${ }^{7,9}$, Belo Horizonte ${ }^{5}$ e um multicêntrico ${ }^{10}$, também foi constatado que o transplante cardíaco foi realizado, em sua maioria (acima de 70\%), em pacientes do sexo masculino.

De acordo com o relatório da Internacional Society for Heart and Lung Transplantation ${ }^{11}$, a ocorrência de transplantes cardíacos em pacientes do sexo masculino foi superior a $70 \%$, no período de 2002 a jun/2009 e ainda vários estudos demonstram que 0 índice de pacientes do sexo masculino submetidos ao transplante cardíaco é superior ao do sexo feminino ${ }^{12}$.

Em relação à média de idade de pacientes submetidos a transplante cardíaco, foi encontrada a média de 54,0 anos ${ }^{11}$, que é superior à do nosso estudo, que foi de 45,2 anos. Outros estudos encontraram dados semelhantes ao nosso: média de 44,8 anos ${ }^{9}$; de 44,9 anos 7 ; de 46,9 anos ${ }^{8}$. Estes resultados mostram que a maioria dos pacientes transplantados estava em idade considerada produtiva.

No relatório do grupo de transplante espanhol de 2006 a taxa de mortalidade nos primeiros 30 dias após o transplante foi de $14,0 \%{ }^{11}$. Em nosso estudo, esta taxa foi de $18,1 \%$, o que é bem acima do que foi considerado alto pelos autores citados, porém semelhante à encontrada em São Paulo $(18,7 \%)^{9} \mathrm{e}$ menor do que a encontrada em Pernambuco $(20,0 \%)^{8}$.

Dos pacientes que compuseram a amostra do presente estudo à época do transplante, $18,1 \%$ já se encontravam aposentados devido à limitação para atividades que a insuficiência cardíaca provoca.

Percebe-se que esse impacto ocorre face ao grande número de pacientes aposentados nessa faixa etária, assim como ao número de internações por descompensação cardíaca. Nesse estudo, 56,8\% dos pacientes estavam internados quando foram chamados para o transplante ou já haviam sido internado, de três vezes a cinco vezes, nos meses anteriores ao transplante.

Com relação ao estado civil, nosso estudo assemelha-se ao outro realizado em Belo Horizonte, no outro centro transplantador ${ }^{5}$, pois em ambos há o predomínio de pacientes casados.

A análise do grau de instrução dos pacientes transplantados cardíacos ficou deficitária devido à ausência de registros em muitos prontuários. Com o início da Sistematização da Assistência de Enfermagem (SAE) nesta instituição, em agosto de 2008, percebemos nitidamente uma melhoria do registro dos dados pela equipe de enfermagem. Mesmo assim, ainda há um grande déficit de informações relacionadas aos anos anteriores à implantação do processo de enfermagem. Dos dados registrados, a grande maioria tem o ensino fundamental $(62,5 \%)$.

A profissão/ocupação, de modo geral, retrata o nível de instrução do paciente. Na amostra estudada, houve 0 predomínio de profissões/ocupações que exigem o ensino fundamental incompleto ou completo $(47,7 \%)$.

Em nosso estudo, percebeu-se que os locais de moradia da grande maioria dos pacientes eram capital e região metropolitana (52,3\%), mantendo um equilíbrio com pacientes residentes do interior de MG $(47,7 \%)$.

Em um estudo realizado ${ }^{5}$ em outro centro transplantador de Belo Horizonte, detectou-se que 73,5\% dos pacientes residiam em BH, número bem superior ao nosso.

Vale ressaltar que mesmo os pacientes que moram no interior do estado devem permanecer durante o transplante e tratamento inicial depois dele próximo ao centro transplantador, ou seja, em BH e RMBH. Esse fato pode trazer dificuldades nos registros, confundindo procedência com moradia. Para evitar tal situação, optamos por colher esse dado registrado na primeira consulta.

Não foi encontrado o registro da religião em $65 \%$ dos prontuários. 0 cuidado prestado ao paciente, principalmente pela enfermagem, deve ser de maneira holística. 0 ser humano deve ser visto com seus valores, crenças e necessidades próprias.

Em um estudo com pacientes em pós-operatório mediato de transplante cardíaco, o diagnóstico de enfermagem de "angústia espiritual" ou o risco de "angústia espiritual" estava presente em 100\% dos pacientes submetidos a esse procedimento ${ }^{5}$. Portanto a falta de informação sobre a religião professada pelo paciente pode influenciar na assistência holística ao ser humano.

Quanto ao perfil epidemiológico, vários estudos apontam as principais etiologias que acometem os pacientes portadores de insuficiência cardíaca irreversível, ou seja, as que levam a um transplante cardíaco.

Estudos realizados em São Paulo mostram que os pacientes transplantados de coração apresentavam como primeira etiologia de base a miocardiopatia dilatada idiopática, seguida da miocardiopatia isquêmica, enquanto a miocardiopatia chagásica apareceu em terceiro lugar ${ }^{7,9,10}$. Em Pernambuco, a miocardiopatia isquêmica aparece em primeiro lugar, seguida da miocardiopatia dilatada idiopática e apenas um paciente com miocardiopatia chagásica ${ }^{8}$. Estes resultados diferem dos nossos achados que apontam a miocardiopatia chagásica como causa da insuficiência cardíaca que levou ao transplante em 50,0\% dos pacientes, seguida pela miocardiopatia dilatada idiopática (20,5\%) e miocardiopatia isquêmica (15,9\%).

Podemos supor que o percentual de pacientes que tiveram uma miocardiopatia chagásica se deve ao fato de 
alguns (13) deles terem nascido ou serem residentes no interior de Minas Gerais, em região endêmica para a doença de Chagas, por exemplo, cidades da região do Vale do Jequitinhonha.

Alguns hábitos de vida, como o tabagismo e o alcoolismo, são prejudiciais à saúde, e, em se tratando de pacientes com cardiopatias, esses hábitos colaboram para o agravamento da doença. 0 tabagismo é um fator predisponente às doenças cardiovasculares ${ }^{13}$. 0 consumo progressivo e continuado de bebida alcoólica contribui para a deterioração progressiva da função miocárdica, agravando a disfunção ventricular $^{14}$.

Assim, os hábitos nocivos à saúde são fatores que causam problemas cardiovasculares e podem comprometer 0 transplante cardíaco a médio e a longo prazo.

Dos pacientes da amostra estudada, 38,6\% eram tabagistas e 43,2\% faziam uso de bebida alcoólica antes da realização da cirurgia. Destaca-se que em $6,9 \%$ prontuários não foram encontrados registros sobre o tabagismo e em 18,2\% prontuários sobre o uso de bebida alcoólica, a falta ou a imprecisão de informações, tais como "consumo socialmente" e "apenas nos fins de semana", não permitiu a identificação do tempo e da quantidade de bebida alcoólica ingerida.

Mesmo esses pacientes sendo obrigados a abandonar esses vícios antes da realização do transplante, é fundamental conhecer o histórico do candidato ao transplante com o intuito de se promover a orientação ou reorientação do paciente e familiares sobre os riscos desses hábitos e apoiá-los para manutenção desse comportamento. Portanto, não há como relacionar esse hábito com doença e as complicações decorrentes dele, tanto no pré como no pós-operatório.

A assistência deve ser prestada ao paciente de forma holística, ou seja, como ser biopsicossocioespiritual. No que tange às necessidades biológicas, essas não podem se restringir apenas à condição que levou o paciente à cirurgia, pois ele pode ter outras patologias associadas, que podem, inclusive, influenciar a evolução do paciente no pós-operatório, propiciando o aparecimento de complicações.

Poucos estudos analisam de forma sistemática a incidência de complicações apresentadas durante o pósoperatório imediato, e autores afirmam que o primeiro mês de pós-operatório de transplante cardíaco é um período crítico devido à alta taxa de morbimortalidade ${ }^{12}$.

Considerando a diversidade das complicações apresentadas, optou-se por agrupá-las quando interrelacionadas. Foram consideradas complicações pós-cirúrgicas aquelas que se relacionam com o ato cirúrgico em si.

Em nosso estudo, o sangramento foi considerado aumentado quando a drenagem era igual ou maior a 4,0 ml/ $\mathrm{kg} / \mathrm{h}$, conforme protocolo da instituição. Essa complicação foi registrada em prontuários de 13 pacientes no primeiro dia de pós-operatório, e todos tiveram indicação de transfusão sanguínea e 6,8\% dos pacientes foram submetidos à revisão cirúrgica para hemostasia, sendo que um deles foi a óbito.
Em um estudo ${ }^{8}$, dos 35 pacientes transplantados de coração, 8,5\% necessitaram ser reoperados devido a sangramento. Esse valor é inferior ao encontrado em outros estudos como no Chile $(16,0 \%)^{15}$.

As causas para ocorrência de sangramento aumentado são multifatoriais. Os resultados de um estudo apontam que a maioria dos pacientes com sangramento aumentado apresentava disfunção hepática prévia ${ }^{16}$. Em nosso estudo, todos os pacientes com tal complicação já haviam sido se submetido ao implante de marca-passo e, posteriormente, entraram na fila de urgência, o que pode significar uma deterioração nos sistemas orgânicos por baixo débito cardíaco e distúrbios de coagulação.

Quanto ao tamponamento cardíaco, em nosso estudo, $15,9 \%$ dos pacientes apresentaram tal complicação, todos eles passaram por revisão cirúrgica e $42,8 \%$ deles faleceram. No estudo realizado na Colômbia, 18,7\% pacientes apresentaram tamponamento ${ }^{16}$, esse achado é superior ao nosso. 0 derrame pericárdico volumoso foi uma complicação apresentada por $15,9 \%$ pacientes. Pouca menção foi feita ao sangramento e tamponamento cardíaco na literatura pesquisada, e nenhuma a derrame pericárdico volumoso.

A rejeição é uma complicação inerente aos pacientes transplantados de órgãos, além de ser uma das principais causas de mortalidade. No pós-operatório imediato, autores relatam que 14,2\% pacientes apresentaram rejeição, sem ocorrência de óbito ${ }^{9}$. Esse valor é maior ao encontrado na Espanha que, em 2005, foi de 10,0\% ${ }^{11}$. Outro estudo encontrou 46,6\%, considerando todos os graus de rejeiçãa $0^{16}$.

Em nosso estudo, contabilizamos a rejeição somente a partir do grau II, uma vez que a rejeição grau I ou discreta é prevista em transplante de órgãos e que não são tomadas medidas terapêuticas, somente vigilância, no hospital campo do estudo. Encontramos 45,5\% de pacientes com rejeições (graus II e III), sendo que metade deles apresentou rejeição grave (grau III). Vale ressaltar que, nos outros estudos citados, não há referência sobre a classificação da rejeição que adotaram para contabilizá-la, o que impede de fazer comparações com os nossos achados. Outro aspecto de nosso estudo que deve ser destacado foi a necessidade de suspensão ou troca do imunossupressor (ciclosporina) para melhora da função renal, pois, a maioria dos pacientes apresentou insuficiência renal. Esse fato talvez possa estar relacionado com a alta taxa de rejeição encontrada nos primeiros 30 dias de pós-operatório.

Em nosso estudo, 36,3\% dos pacientes apresentaram disfunção do enxerto no primeiro mês do transplante, resultado bastante diferente do encontrado em outro estudo ${ }^{16}$, que foi de $8,0 \%$ no mesmo período de pós operatório. Pode-se supor que esta diferença esteja ligada às variáveis transoperatórias e às condições do receptor no pré-operatório, entre outros fatores não avaliados nos estudos.

Infecção foi uma complicação que se manifestou em qualquer período do pós-operatório, predominando as pneumonias $(25,0 \%)$, sinusites $(6,2 \%)$, infecções urinárias 
$(6,2 \%)$ e mediastinite $(4,2 \%)$. Dos 12 pacientes que tiveram pneumonias, 4 morreram. Nosso achado se assemelha a outros estudos ${ }^{10,12}$

Os imunossupressores são responsáveis por vários efeitos indesejáveis, reversíveis em sua maioria, que podem afetar a evolução dos pacientes, entre eles estão: hipertensão arterial sistêmica, dislipidemia, hirsutismo, hiperplasia gengival, ganho de peso e insuficiência renal.

Em nosso estudo, 29,5\% dos pacientes apresentaram hipertensão arterial no pós-operatório imediato. Várias causas podem ser aventadas como o uso de inotrópicos, decorrente da insuficiência renal e de imunossupressores.

Entre as complicações neurológicas, a cefaleia foi a complicação mais incidente tanto no pós-operatório imediato quanto no tardio. Porém, as crises convulsivas foram predominantes no pós-operatório imediato.

A insuficiência renal foi a segunda complicação que mais se manifestou nos pacientes dessa amostra $(59,0 \%)$. Essa complicação foi atribuída ao uso de imunossupressor para 42,3\% dos pacientes e à circulação extracorpórea para 46,1\% . Os outros 3 pacientes desenvolveram tal complicação por baixo débito cardíaco, sendo 2 deles por sepse. Um estudo com pacientes em pós-operatório em unidade de terapia intensiva encontrou $5,3 \%$ dos pacientes com necessidade de hemodiálise ${ }^{15}$. Em nosso estudo, 7,6\% pacientes foram submetidos a esse tipo de terapia.

A alteração glicêmica é comumente observada em pacientes no pós-operatório. Em nosso estudo, 81,8\% apresentaram hiperglicemia no pós-operatório imediato sendo que, na maioria $(79,5 \%)$ deles, esta complicação se manifestou nas primeiras 72 horas da cirurgia. Destaca-se que 3 desses pacientes já eram diabéticos e um necessitou corrigir a hiperglicemia com insulinoterapia administrada continuamente. 0 paciente que apresentou hiperglicemia no $18^{\circ} \mathrm{DPO}$ estava com corticosteroide endovenoso, o que pode explicar a hiperglicemia em pós-operatório mais tardiamente.

Os imunossupressores inibidores de calcineurina (ciclosporina e tacrolimus) associados aos corticosteroides aumentam a intolerância à insulina e podem causar a diabetes mellitus, principalmente, 0 tacrolimus ${ }^{11}$.

Verifica-se que as complicações encontradas nos pacientes submetidos ao transplante cardíaco são multifatoriais, além de serem muitas. No âmbito da enfermagem, cabe reconhecer os sinais e sistematizar aç̃̃es voltadas para o cuidado de forma holística.

\section{CONSIDERAÇÕES FINAIS}

Tendo em vista os objetivos propostos chegamos às seguintes conclusões: A maioria dos pacientes que se submeteram ao transplante cardíaco era do sexo masculino, casada, escolaridade de nível fundamental e tinha profissão/ ocupação que exigia este grau de instrução. A idade variou de 21 a 64 anos (média de 45,2 anos). Todos residiam em Minas
Gerais, sendo a grande parte em Belo Horizonte ou região metropolitana.

A miocardiopatia chagásica foi a causa de insuficiência cardíaca em metade dos pacientes, seguida pela miocardiopatia dilatada idiopática. As complicações que mais acometeram os pacientes foram: insuficiência renal, infecções, disfunção do enxerto cardíaco, disfunções gastrointestinais, sangramento aumentado, cefaleia, arritmias, choque, sepse, tamponamento, derrame pericárdico, convulsões e encefalopatia.

Não foram encontrados registros de complicações relacionadas às necessidades psicossociais e psicoespirituais, com exceção de ansiedade e depressão. 0 aprofundamento de questões ligadas ao transplante cardíaco é fundamental para o desenvolvimento científico da área, para prestação da assistência de forma holística e humanizada, visando desfechos cada vez mais favoráveis.

Esperamos que os resultados desse estudo subsidiem reflexões dos profissionais e contribuam para a história dos transplantes de coração no hospital campo do estudo e de Minas Gerais. Realizar um trabalho científico exige transpor obstáculos e conviver com limitações, em vários aspectos. Desenvolver essa pesquisa foi um desafio decorrente de vários fatores. 0 principal deles foi a coleta de dados nos prontuários ainda impressos em vários volumes e com registros muitas vezes ilegíveis. As questões não respondidas nesse trabalho e as suscitadas por ele devem ser motivos de novos estudos. Fazse necessária a realização de pesquisas com ênfase nos aspectos psicossociais e espirituais, uma vez que o ser humano deve ser visto como ser holístico.

\section{REFERÊNCIAS}

1 World Health Organization-WHO. Preventing chronic disease: a vital investment.WHO Global Report. Geneva; 2005.

2 Ischitani LH, Franco GC, Perpétuo IHO, França E. Desigualdade social e mortalidade precoce por doenças cardiovasculares no Brasil. Rev Saude Publica. 2006; 40(4): 684-91.

3 Ministério da Saúde (BR). Saúde Brasil 2007: uma análise da situação de saúde. Brasília (DF); 2008.

4 Fiorelli Al, Coelho GHB, Junior JLO, Oliveira AS. Insuficiência cardíaca e transplante cardíaco. Rev Medica. 2008 abr/jun; 87(2): 105-20.

5 Matos SS. Diagnósticos de enfermagem em pacientes no pós-operatório de transplante cardíaco e validação do diagnóstico considerado mais característico [tese]. Belo Horizonte: Escola de Enfermagem, Universidade Federal de Minas Gerais; 2009.

6 Carvalho DV, Salviano MEM, Carneiro RA, Santos FMM. Diagnóstico de enfermagem de pacientes em pós-operatório de transplante hepático por cirrose etílica e não-etilica. Esc Anna Nery. 2007 out/dez; 11(4): 682-87. 
7 Moraes Neto F, Tenório D, Gomes CA, Tenório E, Hazin S, Magalhães M, et al. Transplante cardíaco: a experiência do Instituto do Coração de Pernambuco com 35 casos. Rev Bras Cir Cardiovasc. 2001 abr/jun; 16(2): 152-59.

8 Branco JNR, Teles CA, Aguiar LF, Vargas GF, Hossne Junior MA, Andrade JCS, et al. Transplante cardíaco ortotópico: experiência na Universidade Federal de São Paulo. Rev Bras Cir Cardiovasc. 1998 out/dez; 13(4): 285-94.

9 Assef MAS, Valbuena PF, Neves Junior MT, Correia EB, Vasconcelos M, Manrique R, et al. Transplante cardíaco no Instituto Dante Pazzanese de Cardiologia: análise da sobrevida. Rev Bras Cir Cardiovasc. 2001 out/dez; 16(4): 289-304.

10 Bocchi EA, Fiorelli, A. The brazilian experience with heart transplantation: a multicenter report. J Heart Lung Transplant. 2001; 20(6): 637-45.

11 Stehlik J, Edwards LB, Kucheryavaya AY, Aurora P, Christie JD, Kirk R. et al. The Registry of the International Society for Heart and Lung Transplantation: Twenty-Seventh Official Adult Heart Transplant Report2010. J Heart Lung Transplant. 2009; 29(10): 1089-103.

12 Jáuregui I, Ramón-Llin JA, Bonet A, Rubio AV, Marín JP, Martínez-Dolz L. Incidencia y predictores de complicaciones graves trás el transplante cardíaco en la unidad de reanimación. Rev Esp Anestesiol Reanim. 2008; 55: 535-40.

13 Bocchi EA, Marcondes-Braga FG, Ayub-Ferreira SM, Rohde LE, Oliveira WA, Almeida DR, et al. Sociedade Brasileira de Cardiologia. III Diretriz Brasileira de Insuficiência Cardíaca Crônica. Arq Bras Cardiol. 2009; 93(1 supl 1): 1-71.

14 Gonçalves AM, Correia AM, Falcão LM, Ravara AL. Miocardiopatia alcoólica: significado clínico e prognóstico. Rev Soc Port Med Int.[online] 2005 abr/jun; [citado 2011 maio 10]; 12(2): [aprox 12 telas]. Disponível em: http://www.spmi.pt

15 Aranguiz-Santander E, Merello L, Pedemonte OP, Torres H, Vera A, Alburquerque J. Heart transplantation in Chile: preliminary report from the Gustavo Fricke Hospital in Vina del Mar. Transplant Proc. 2007 Apr; 39(3): 619-21.

16 Jamarilho JC, Villegas A, Fernández D, Ramirez A, Durán M, Montoya M. et al. Función de riesgo para la supervivencia en pacientes com trasplante cardíaco. Rev Colomb Cardiol. 2007 ene/feb; 14(1): 33-42. 